\title{
MOLECULAR SPECTROSCOPY
}

$\mathrm{T}$ HE ramifications of molecular spectroscopy in pure and applied physies and chemistry are now so numerous and technical progress is so rapid that frequent conferences about them are not superfluous. By any standard, the two-day meeting arranged during October 28-29 by the Hydrocarbon Research Group of the Institute of Petroleum was a success. By bringing together academic and industrial workers in Britain, and others from several European countries and the United States, a critical review of current problems was possible, and future trends in technique and theory were considered. The topics discussed ranged from the vacuum ultra-violet to the far infrared, and although many papers were directed towards hydrocarbon chemistry, there was a balanced inclusion of results on a wider scale. So far as analytical work and structural diagnosis are concerned, it is becoming increasingly important to choose the most appropriate of the various physico-chemical methods. A similar choice may now be made between the various spectroscopic methods themselves, which are so often not alternative but supplementary to each other.

Opening the first session, E. J. Bowen (Oxford) made a critical evaluation of fluorescence measurements for industrial analysis and examination. Promising as this phenomenon might appear for the study of aromatic or heterocyclic compounds, great care is needed in its interpretation. Older data are often misleading, for fow compounds fluoresce, and the fluorescence is destroyed or affected by trace compounds or other factors. Purity of a solvent, and the method of excitation and of spectral recording are important, and re-absorption of the fluorescence must be considered. There may be a gain in the analysis of oils by prior chromatography, or by lowtemperature measurements in a solvent which congeals to a clear glass. Yet more information is required about such processes as 'resonance transfer' whereby one molecule transfers its energy of excitation to another trace compound and induces a fluorescence which may be entirely misleading.

G. Porter and M. W. Windsor (Cambridge) described results of another kind on the fluorescence and phosphorescence of aromatic hydrocarbons. It is known that, when suitably irradiated, as solids in glassy solvents, these substances show, in addition to the normal fluorescence which appears during illumination, a slower re-emission of radiation; the latter is composite, one part having a wave-length identical to the main fluorescence and a temperaturedependent decay, the other being a slower phosphorescence of longer wave-lengths. This phosphorescence has been interpreted in terms of a metastable triplet electronic state into which the molecules pass after initial excitation. By means of their elegant method of flash photolysis, Porter and Windsor have now obtained absorption spectra due to the transitions between the triplet levels for solutions of the hydrocarbons. Some microseconds after the flash, while enough molecules are still in the lowest triplet state, an absorption spectrum is measured, and by adjustment of the time interval the decay of the triplet level has been studied from the absorption-band intensities. From the remarkable effect of the solvent viscosity on the kinetics of the decay process, the nature of the triplet state has been examined. The results obtained by this novel technique may have an important bearing upon the photochemistry of aromatic hydrocarbons and of dyestuffs.

B. F. Dudenbostel (Standard Oil Development Co.) reviewed the present state of Raman spectroscopy. As a result of the Toronto mercury arc, the magnesium oxide reflector and more sensitive uncooled electron multiplier tubes, photoelectric recording appears to have replaced photography. Samples as small as $1 \mathrm{ml}$. can be used, but suspended dust or traces of fluorescent materials must be avoided. The main advantage of direct recording lies, of course, in the direct determination of line intensities or of integrated areas under the recorded peaks. No generalization can be made about which analytical problems will be more suited to Raman or infra-red work, but the former may find much use with aromaties, especially, perhaps, in the higher-boiling petroleum fractions and also with certain mixtures of olefins. The development of an intense monochromatic source of somewhat longer wave-length, with an appropriately sensitive detector, might extend this work considerably.

A vacuum grating spectrometer with photoelectric recording to $1700 \mathrm{~A}$. was described by R. A. Burdett, L. W. Taylor and L. C. Jones (Shell Oil Co.). An E.M.I. photomultiplier with a thin (1-mm.) quartz window was used. A number of substituted benzenes, polynuclear aromatic hydrocarbons and sulphur compounds dissolved in heptane have characteristically different spectra which can be used for analysis more effectively than those in the normal ultra. violet, especially if some preliminary chromatography is carried out. It would seem that a grating is preferable to a prism in the far ultra-violet and that a better source may be obtained for the region 2100 $1500 \mathrm{~A}$. by excitation of xenon with a microwave discharge.

The second session dealt with infra-red technique. V. Z. Williams (Perkin-Elmer Corporation) discussed critically the instruments now available for research and for industrial analytical control. The choice between different spectrometers is now mainly governed by the nature of the problem under investigation. Single-beam instruments, with interchangeable prisms covering a wide spectral range, and a double-pass system to increase resolving power and also to eliminate false energy, have high photometric a.ccuracy and elasticity as regards use with accessories such as the reflecting microscope or long-path absorption cells. Double-beam spectrometers, on the other hand, produce a direct spectral record and eliminate atmospheric absorption, and for routine qualitative work in organic chemistry these factors are important. Also, differential analysis using the double-beam arrangement shows much promise for analytical work. However, the flexibility of doublebeam instruments is more limited and their photometric accuracy may be less. For pilot-plant or 
process control, continuously operating infra-red analysers are now in use for determining common gases in fairly complex mixtures. Some incorporate a small monochromator, whereas others work by non-dispersive principles. Much attention has been given to their long-term stability under rugged conditions. Their sensitivity can be as high as $0 \cdot 25$ p.p.m. In Dr. Williams's view, "to some extent to-day and more certainly in three years, these instruments will have a potential for the field which will be greater than the ability of the field to utilize".

These spectrometric developments do not necessarily imply an increase in instrumental complexity. In fact, there is a trend towards simplification for much of the work. One leading factor is the replace ment of prisms by cheap echelette diffraction gratings, and L. A. Sayce and A. Jackson (National Physical Laboratory) described their recent work on replication from a helical matrix cut to high accuracy using the method of the Merton nut. The chief experimenta inconvenience of gratings hitherto has been the need for some fore-prism arrangement to eliminate unwanted spectral orders. This difficulty may be removed by use of filters which cut off radiation at the shorter wave-length end. Important advances have occurred here by the discovery that thin layers of silicon, germanium, lead sulphide, coated silver chloride, and other substances have fairly sharp cutoff wave-lengths. More exciting possibilities are suggested by the $F$-centre filters developed by $J$. Gaunt and W. G. Burns (Harwell). Pohl first showed that when crystals of alkali halides acquire a stoichiometric excess of alkali metal they become coloured as a result of trapping of electrons at vacant lattice sites. Such colour centres can be formed by photochemical, radiochemical or electrolytic methods, but more satisfactory results appear to be obtained by heating the crystals with alkali metals. The conditions of preparation and subsequent cutting and polishing still need further study, but good filters appropriate to all the usual spectral regions have been produced. Their additional value for eliminating false radiation in prism spectrometers at longer wavelengths is also important. The use of crsium iodide as a prism material (extending to $52 \mu$ ) was described by E. K. Plyler (U.S. National Bureau of Standards), and it can be used as a thin window $(2 \mathrm{~mm}$.) out to $65 \mu$. Its dispersive characteristics from $25 \mu$ to $35 \mu$ are not quite so good as those of cæsium bromide. In fact, all spectral needs for a prism spectrometer in the range $2-40 \mu$ can perhaps now be met by lithium fluoride, rock salt and cæsium bromide.

The pressed disk technique used by Stimson and by Schiedt, in which solids are suspended in sintered plates of alkali halide crystal, is improving rapidly. It reduces scatter of radiation, gives sharper absorption bands, eliminates the Christiansen effect and avoids absorption by any mulling agent. M. A. Ford, G. R. Wilkinson and W. C. Price (King's College, London) described their work on this subject and indicated factors such as particle size or sintering pressure which lead to satisfactory disk formation. Care is needed because of spurious impurity bands or possible slow chemical reactions of the solids, and, before quantitative intensity work can be regarded as reliable, further tests are required. T. S. Robinson and W. C. Price (King's College, London) also described a method for calculating the absorption spectrum of a solid from its reflexion spectrum. The computation is rather elaborate, but may be useful in those cases where the absorption of thin films is still too high, as with polytetrafluoroethylene. If polarized radiation is employed the reflectance data may also be used to determine the directions of certain bonds in a molecule with respect to the crystal axes.

Several papers dealt with the interpretation of vibrational spectra for analysis, vibrational assignment and structural diagnosis. Hitherto, infra-red analysis has usually been made on the assumption of additivity of optical densities at band peaks, using extinction coefficients defined by Beer's law. This method is in reality semi-empirical, since it assumes the use of infinitely narrow slit-widths which pass monochromatic radiation. The extinction coefficients depend markedly upon the spectrometer slit-widths unless the latter are small compared with the band half-widths, and this factor alone may account for our inability to transfer infra-red data from one instrument to another. Integrated band areas have a more absolute significance, especially if they are suitably corrected for the spectrometer characteristics so as to yield the true band intensities. The band areas can be computed in several ways, but there is still uncertainty about the exact function which reproduces the band envelopes. Quite apart from their use for analysis, however, band intensity data may lead to further refinement in structural group identification, and since the intensity is related to the variation of molecular dipole during vibration, they may also tell us something about the bond polar properties. These several aspects of work on band intensities were discussed by $\mathrm{H}$. W. Thompson (Oxford) and illustrated by a collection of data so far available. The construction of simple automatic devices for the integration of band areas during spectral measurement will not be long delayed. Whether, owing to band overlap or for other reasons, the true intensities will prove more valuable than extinction coefficients determined with higher spectral resolving-power, is at present an open question.

Tables of molecular vibration frequencies of substituted benzenes, which appear to depend only upon the positions of the substituent groups and not upon their nature, have been collected together by $R$. $R$. Randle and D. H. Whiffen (Birmingham). While there may still be doubt about some of the assignments to specific normal modes, and some of the rules may fail with other substituents, these correlations will be valuable, especially in hydrocarbon chemistry. Similar data were listed by E. D. Kunst (Royal Dutch Shell, Amsterdam) for deutero-aromatics in the region 12-14 $\mu$. H. Lüther (Technical High School, Brunswick) described spectral measurements designed to elucidate the mechanism of oxidation of hydrocarbon oils, using key bands for detection of hydroxyl, ketonic and other groups.

The comparison of vibrational spectra of molecules in different states of aggregation is now leading to important information. This was fully surveyed by N. Sheppard (Cambridge) with particular reference to measurements at low temperatures. The spectral variations observed, and their interpretation, vary according as the molecule is rigid or flexible and also depend upon the coupling of vibrations in the solid lattice. Conclusions have been drawn about the relative stability of rotational isomers and about the assignment of vibration frequencies of long-chain hydrocarbons. In solid nitric acid hydrate there is spectroscopic evidence for the occurrence of the 
$\mathrm{OH}_{3}+$ ion. The sharpening of bands at low temperatures and other features may find application in certain analytical work; but this may involve some experimental problems, and the formation on solidification of an amorphous glass rather than a crystalline mass may be vital.

Attempts to study reaction intermediates and radicals through their spectra have not, so far, been entirely successful. D. F. Hornig (Brown University) described a new approach in which shock waves have been used to excite molecular species to a 'temperature' equivalent to that of reactions or combustion processes. The time required to induce this excitation--some microseconds-may be much less than the reaction times, and it is hoped, therefore, from spectral emission or absorption to study the subsequent kinetic behaviour of the species. Very fast photographic and photoelectric methods have been used to record spectra of certain radicals produced by passing shock waves into substances such as cyanogen halides or methyl halides, and the progress of such work should be interesting. The identification of radicals in flames by resolution of their vibrationalrotational emission bands was achieved recently by Plyler, Benedict and Silverman. G. R. Wilkinson, M. A. Ford and W. C. Price (King's College, London) have now made similar measurements on emission bands in the region 1-5.5 $\mu$ of simple molecules and radicals excited by a radio-frequency discharge in hydrogen halides, methane, water vapour and other gases. This method has definite advantages over absorption measurements in that band sequences are more fully developed, and both the rotational and vibrational constants can be measured conveniently. The results of recent absorption studies on a number of hydrocarbons using very high resolving-power were also outlined by $\mathbf{H}$. W. Thompson. From all these data, more precise values are being obtained, not only for the main molecular rotational constants but also for the centrifugal stretching-coefficients and anharmonic vibration factors.

Among all the above fairly conventional themes, a new note was struck at the meeting - the study of nuclear magnetic resonance and R. E. Richards (Oxford) illustrated its value by many examples of practical importance. The spin of a charged nucleus gives rise to a magnetic effect, and the associated magnetic vector can be oriented in different ways in an applied magnetic field. These orientations correspond to different quantized energy-levels, between which transitions can take place. For fields of 10 kilogauss the absorption frequency lies in the radio region, 'resonance' occurring between the two levels and different nuclei absorbing at characteristically different frequencies. - When interaction occurs between the nuclei and their surroundingsto be exact between their respective magnetic effects -the absorption lines broaden in ways which depend upon the internuclear distances and other structural features. Thus groups such as $\mathrm{CH}_{3}, \mathrm{CH}_{2}$ or $\mathrm{OH}$ can be characterized. Information of the most varied kind, both qualitative and quantitative, can now bo obtained, and it is certain that much will result from this new highly sensitive method.

It is clear that there is intense activity in this field. The difficult task of setting up a punched-card system for reference spectra was also discussed, and it is hoped that the committee set up several years ago in Britain to consider this matter may shortly be able to institute a scheme.

H. W. Thompson

\section{FULMER RESEARCH INSTITUTE, STOKE POGES OPEN DAY}

$\mathrm{A}$ $\mathrm{N}$ informal visit was made to the Fulmer Research Institute on November 2 by H.R.H. the Duke of Edinburgh on the occasion of an open day held to mark the opening of the new Mechanical Testing Laboratory of the Institute. After a tour of the laboratories lasting for an hour and a half, during which he pulled the first test-piece in the new 50-ton tensile-testing machine of the Laboratory, the Duke had luncheon at the Institute together with some three hundred guests representing industry and research. In his speech he said that he had always been convinced that the research business was a good investment and that he was interested to have practical proof in an establishment which, without outside support, does indeed make a profit entirely on research. After luncheon the laboratories were open to the other guests, and the scientific exhibits showed in detail some of the work of the Institute during its first seven years of existence.

Following the invention of the catalytic distillation process for refining of aluminium, Dr. P. Gross and his colleagues demonstrated similar studies applied to the extraction of titanium from impure titaniumbearing materials. Laboratory exhibits showed that titanium can be removed from an impure titaniumbearing alloy by reaction with a high-valency halide to form a halide of lower valency in the vapour phase. Disproportionation of the lower-valency halide then gives a deposit of pure metallic titanium with reformation of the higher-valency halide. In connexion with the work on the purification of both aluminium and titanium, an intensive study is in progress of the reactions which may be involved in these processes, and an important feature of the work is the determination of the heats of formation of metallic halides. The apparatus, which includes dry boxes for handling deliquescent halides and a calorimeter made in the laboratory workshops, was on view. The heat of formation of aluminium fluoride has recently been re-determined by Dr. Gross, and a new value, differing appreciably from that previously accepted, has been established and the results published. The heat of formation of titanium tetrachloride has also recently been determined, but the results obtained are at present confidential to the sponsor. Further work is in hand on the heats of formation of other halides. A recent step forward in Dr. A. H. Sully's work on chromium and its alloys has been the preparation of a ductile form of chromium. Flakes of electrodeposited chromium which are capable of being bent were on view.

In the Physics Section apparatus was set up for studying by $\mathrm{X}$-ray diffraction the structure of liquid metals. This involved the use of Geiger counters in plotting the variation in intensity of $\mathrm{X}$-ray diffraction from a liquid metal surface. Apparatus for measuring the vapour pressure of reactive metals such as uranium was also in operation. Many novel types of camera and a curved quartz erystal monochromator were included in the apparatus for studying the progress of precipitation hardening and the ensuing structural changes. There was an extensive exhibit of the results of such an investigation on the ageing of aluminium copper alloys.

Dr. H. K. Hardy's studies on the thermodynamics of alloy systems and the mechanism of precipitation 